\title{
Stable, coherent free-electron laser pulses using echo-enabled harmonic generation
}

\author{
G. Penn \\ Lawrence Berkeley National Laboratory, Berkeley, California 94720, USA
}

(Received 7 December 2013; published 25 November 2014)

\begin{abstract}
Beam lines using echo-enabled harmonic generation can be designed to have extremely low sensitivity to energy chirps in the electron beam, as shown through theory and detailed simulations. These designs would allow stable and coherent radiation to be produced even when using electron beams with a large amount of shot-to-shot jitter in the longitudinal profile.
\end{abstract}

DOI: 10.1103/PhysRevSTAB.17.110707

PACS numbers: 41.60.Cr, 42.65.Ky

\section{MOTIVATION}

A major motivation for seeding a free-electron laser (FEL) with external lasers is to achieve stable output with coherent pulses having a time-bandwidth product close to the transform limit. While the quality of the seed laser itself is important, any nonuniformity in the electron bunch can also lead to variations in both the power and phase of the output pulse. Gradients in the local average (slice) electron energy are particularly deleterious to output mode quality, especially when a high harmonic of the initial seed laser is desired. Harmonic generation typically requires a dispersive element such as a chicane which shifts different parts of the beam by a distance proportional to the local energy offset. An initially coherent signal can become highly degraded in quality after a combination of longitudinal dispersion and harmonic upshifting.

Echo-enabled harmonic generation (EEHG) [1] uses phase mixing to combine the energy modulations from two input lasers with wavelengths $\lambda_{1}$ and $\lambda_{2}$, producing bunching at a wavelength $\lambda_{X}$. The corresponding wave numbers $k \equiv 2 \pi / \lambda$ are related by $k_{X}=p k_{2}-m k_{1}$, where $p$ and $m$ are integers. Sign conventions are chosen so $p$ and $m$ are typically positive. A chicane is placed after each modulating undulator with dispersion $R_{1}$ and $R_{2}$. The convention used here is that drifts and simple chicanes have dispersion $R_{56}>0$.

The EEHG scheme is inherently less sensitive than high gain harmonic generation (HGHG) [2] to energy chirps in the electron beam $[3,4]$. Through careful adjustment of the parameters for EEHG, this sensitivity will be further reduced to the point where slice energy variations comparable to the FEL bandwidth itself introduce only modest phase errors. Following this prescription allows even an

\footnotetext{
gepenn@lbl.gov

Published by the American Physical Society under the terms of the Creative Commons Attribution 3.0 License. Further distribution of this work must maintain attribution to the author $(s)$ and the published article's title, journal citation, and DOI.
}

electron beam with large energy chirps that fluctuate from shot to shot to generate stable and coherent radiation. The technique of coupling multiple inputs through phase mixing is used in other fields such as magnetic resonance and plasma physics. The added flexibility from allowing for reduced coupling could be useful in a wide range of systems to enhance stability or coherence.

\section{ANALYSIS}

In the HGHG scheme, microbunches experience a longitudinal displacement of roughly $\Delta z \simeq R_{56} \bar{\eta}$ as they pass through a chicane, where $\bar{\eta}$ is the relative offset of the average electron energy in the microbunch from the nominal energy. These displacements change the phase of the complex bunching parameter, defined as $\hat{b} \equiv$ $\left\langle\exp \left(-i h k_{1} z\right)\right\rangle$ at the harmonic $h$ of an input laser with wavelength $\lambda_{1}$. The resulting phase shift is given by

$$
\Delta \Psi \simeq-h k_{1} R_{56} \bar{\eta} .
$$

The quantity $\Delta \Psi$ will also be the local shift in phase of the initial radiation produced in a downstream undulator. Note that the shorter the final wavelength, the greater impact a given $R_{56}$ will have on the coherence.

In the EEHG scheme tuned to produce bunching at a wavelength $\lambda_{X}$, neglecting for the moment the effect of energy scattering due to incoherent synchrotron radia tion (ISR) and intrabeam scattering (IBS), the local complex bunching parameter $\hat{b} \equiv\left\langle\exp \left(-i k_{X} z\right)\right\rangle \quad$ is approximately [5]

$$
\hat{b} \simeq \mathrm{e}^{i \Psi_{L}} J_{p}\left(C_{2} \eta_{M 2}\right) J_{m}\left(C_{1} \eta_{M 1}\right) \int \mathrm{d} \eta f_{\eta}(\eta) \mathrm{e}^{-i C_{1} \eta} .
$$

Here, $J_{m}$ and $J_{p}$ are Bessel functions, $C_{1} \equiv k_{X} R_{2}-m k_{1} R_{1}$, $C_{2}=k_{X} R_{2}$, the two modulating undulators produce fractional energy modulations $\eta_{M 1}$ and $\eta_{M 2}$, and it is assumed that only one set of values for $m$ and $p$ is significant. In the integral, $\eta$ is the fractional energy offset from the nominal energy and $f_{\eta}$ is the local energy distribution function. 
The laser phase term $\Psi_{L} \equiv p\left(\pi+\theta_{2}\right)-m \theta_{1}$ combines the local phases of the two seed lasers. Note that typically $p \gg$ $m$ and the overall phase is much more sensitive to the phase of the second laser than to that of the first laser. The magnitude of the bunching is then much more sensitive to $C_{2} \eta_{M 2}$ than it is to $C_{1} \eta_{M 1}$. For a Gaussian energy distribution,

$$
\int \mathrm{d} \eta f_{\eta}(\eta) \mathrm{e}^{-i C_{1} \eta}=\mathrm{e}^{-i C_{1} \bar{\eta}} \mathrm{e}^{-C_{1}^{2} \sigma_{\eta}^{2} / 2},
$$

where $\sigma_{\eta}$ is the local (slice) energy spread. Note that the same term, $C_{1}$, describes both the sensitivity to energy chirps (through $C_{1} \bar{\eta}$ ) and to the energy spread (through $C_{1}^{2} \sigma_{\eta}^{2}$ ). Scattering will reduce the bunching but does not affect the phase terms $[6,7]$. Thus we obtain

$$
\Delta \Psi \simeq-C_{1} \bar{\eta}=-k_{X}\left(R_{2}-m R_{1} \lambda_{X} / \lambda_{1}\right) \bar{\eta} .
$$

In analogy with the HGHG result, this leads us to define an apparent dispersion

$$
R_{\text {echo }} \equiv R_{2}-m R_{1} \lambda_{X} / \lambda_{1}
$$

Higher values of $R_{\text {echo }}$ imply more sensitivity to distortions in the longitudinal phase space of the electron beam. Maximum bunching occurs by independently optimizing $C_{1}$ and $C_{2}$ to maximize the Bessel function terms. Then the ratio $\left|R_{\text {echo }} / R_{2}\right|=\left|C_{1} / C_{2}\right| \sim m \eta_{M 2} / p \eta_{M 1}$, which is usually small when $\lambda_{X} \ll \lambda_{1}, \lambda_{2}$. Typically, it is only necessary to generate bunching above a certain level, for example to overwhelm shot noise, and it is not necessary to tune the EEHG scheme for maximum bunching. By changing either $R_{1}$ or $R_{2}$ the value of $R_{\text {echo }}$ can be easily changed, at the possible cost of increasing the distance needed to reach saturation. However, if $R_{\text {echo }}$ is set to 0 , then the argument of $J_{m}$ is set to 0 and no bunching is generated.

One underappreciated aspect of the EEHG scheme is that there are two configurations which maximize the bunching $|\hat{b}|$ : one where $R_{\text {echo }}$ and thus the argument of $J_{m}$ is positive and one where it is negative. Scanning in $R_{1}$ will yield a double peak in the bunching parameter, with the bunching dropping to 0 in between. The peak corresponding to the larger value of $R_{1}$ will have negative $R_{\text {echo }}$, which means that the EEHG stage acts as if it were composed of chicanes having the opposite sign dispersion from what they actually are. This effect has already been noted in Ref. [8], where the variation of output wavelength with electron energy chirp for a specific set of EEHG parameters has the opposite sign as that for an HGHG beam line. The same effect can be observed below by comparing the phase profiles in Fig. 5b and Fig. 8. However, the authors do not take into account the fact that subsequent radiation stages will begin to reverse this chirp. Moreover, the condition that $C_{1}=0$ is not identified as the point at which the bunching goes to zero due to the $J_{m}$ term. For the typical setting where $m=1$, the bunching parameter will increase roughly linearly with the sensitivity to energy spread and energy chirps until the bunching approaches its peak value.

It may seem strange that the bunching phase can shift in the reverse direction from the motion of individual electrons. To illustrate the mechanism, Fig. 1 portrays the longitudinal phase space at various stages during EEHG starting from a top-hat energy distribution function.
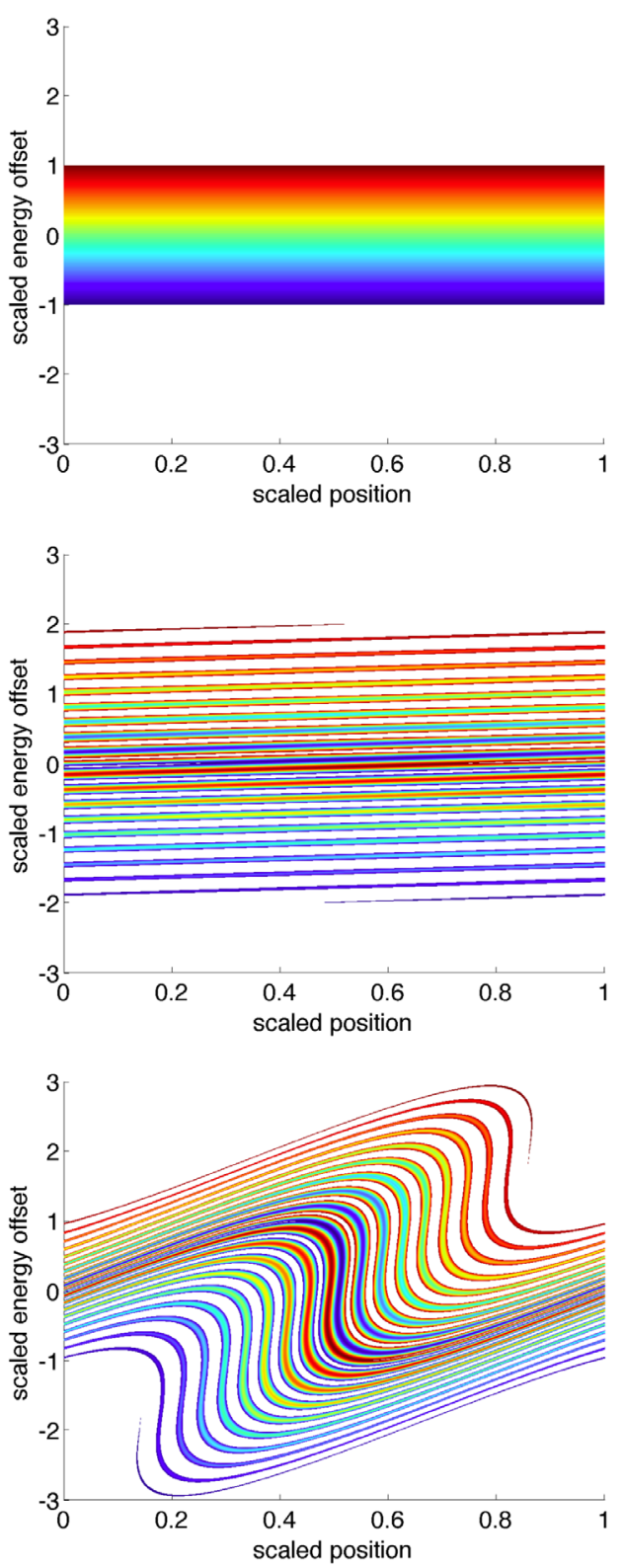

FIG. 1. Phase space distributions throughout the EEHG manipulations. The original beam (top) was a flat-top in energy between -1 and +1 in scaled co-ordinates. After the first modulation and chicane the beam is overbunched (middle). The final distribution (bottom) has maximum bunching at the 25th harmonic, but the typical spacing between adjacent bunches is close to the 27 th harmonic. Incoherent radiation and scattering have been ignored in these figures. 
The beam manipulations are similar to those in the simulations below. The overall bunching is optimized for the 25th harmonic with $R_{\text {echo }}<0$, but individual microbunches are spaced closer together with a separation closer to the 27th harmonic and there is a dislocation in the center. The phase space is color-coded by the original energy offset: blue for electrons with the lowest initial energy, red for the highest initial energy. If the energy at one location in the bunch is increased, the microbunch furthest to the left will begin to vanish and a new microbunch will appear on the right. In terms of the bunching modulo the harmonic wavelength, this corresponds to a shift to the left. The variation of the bunching parameter from performing a scan in $R_{1}$ is shown in Fig. 2. The impact of scattering has been ignored in both figures.

The first stage produces a typical stretch-and-fold pattern of mixing, where in this case the core phase space of the beam is mixed with regions of lower phase space density which were originally situated at high and low energies. Because of the large amount of interleaving between occupied and unoccupied regions of phase space, the energy spread only has to be doubled at the end of the first stage in order to produce significant bunching at the 25th harmonic in the final distribution. On the other hand, the narrow separation between energy bands in the intermediate stage makes EEHG sensitive to small amounts of energy scatter, for example due to IBS and ISR.

It is advantageous to choose $R_{\text {echo }}<0$, because then the initial phase errors at the end of the EEHG stage will be slowly reversed by dispersion further downstream. If the original phase errors when tuned for maximum bunching are too large to be eliminated by the downstream portion of the beam line, then $R_{1}$ can be decreased to reduce the initial phase errors at the cost of reduced bunching. This will incidentally reduce the sensitivity to energy spread at the same time. The total undulator length required to reach saturation will then increase, which also helps to achieve cancellation of the final phase errors. This is the case in the

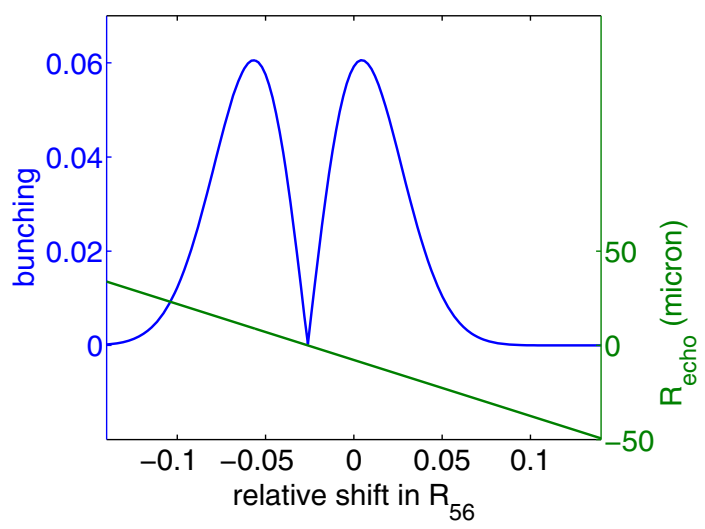

FIG. 2. Dependence of the magnitude of the bunching parameter as the value of $R_{1}$ is varied. All other parameters are kept fixed. Incoherent radiation and scattering have been ignored. examples described below. If instead the downstream dispersion dominates over the initial phase offsets, the value of $R_{1}$ must be increased (which will then increase the sensitivity to energy spread). Note that $R_{2}$ could be adjusted instead of $R_{1}$. This also changes the value of $J_{p}$, but the impact is usually inconsequential.

In principle, it should be possible to tune for a completely flat radiation phase in the output pulse, regardless of the initial longitudinal phase space profile. In practice, there are several ways in which this may fail. When the slice energy offset is comparable to the FEL bandwidth, the power drops and nonlinear dependencies on the energy offset become large. When there are rapid chirps in the slice energy, the effect of slippage disrupts the relationship between the radiation phase and the local energy offset. Longitudinal wakefields such as those from coherent synchrotron radiation (CSR), longitudinal space charge, or interactions with the beam pipe will result in phase errors that are uncompensated because the slice energy has changed.

Variations of the input laser are another potential source of phase variations or power fluctuations. As seen in Eq. (2), when $p \approx \lambda_{2} / \lambda_{X} \gg 1$ the bunching produced by EEHG is highly sensitive to the instantaneous fields of the second laser [9]. Variations in the phase of the input laser are multiplied by a factor $p$ in the bunching phase. Similarly, because of the Bessel function term $J_{p}$, the magnitude of the bunching parameter is highly sensitive to both the peak intensity and to transverse gradients in the intensity. The first peak of the Bessel function scales with $p$ while the width of the peak scales as $p^{1 / 3}$. The energy modulation scales as the square root of the intensity, and so relative variations in the intensity must be much smaller than $p^{-2 / 3}$. This causes the duration of the output pulse to be shorter than that of the input laser by a factor of roughly $p^{-1 / 3}$ for a Gaussian profile, and requires careful control of the laser phase and peak power as well as a large spot size to ensure uniformity. For $\lambda_{2} / \lambda_{X}=25$, as considered here, relative intensity fluctuations must be significantly smaller than $10 \%$. Slippage could actually help both of these effects by smoothing out short-scale fluctuations, but large-scale nonlinearities in the phase tend to be a larger problem than sidebands. Shot-to-shot stability of the laser power will have to be tightly constrained. When further upshifting the radiation to the 100th harmonic, chirps in the laser phase will be multiplied by a factor of 100 overall. This enhanced sensitivity to input phase applies to HGHG as well.

\section{SIMULATION RESULTS}

We consider the three beam lines shown in Fig. 3. The seed lasers all have a wavelength of $200 \mathrm{~nm}$. The average beta function is $10 \mathrm{~m}$ in each beam line. The HGHG beam line uses a seed laser power of $600 \mathrm{MW}$, and radiates at the 10th harmonic $(20 \mathrm{~nm})$. The EEHG beam line uses two identical seed lasers with $43 \mathrm{MW}$ peak power, and radiates 


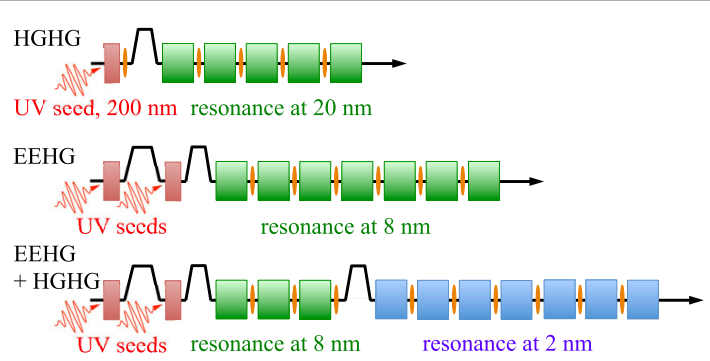

FIG. 3. Beam line layouts using HGHG, EEHG, and a combination of both. The thin ellipses indicate quadrupoles.

at the 25th harmonic $(8 \mathrm{~nm})$. The combined EEHG and HGHG beam line produces radiation at the 25th harmonic in the same configuration, followed by an additional harmonic jump to $2 \mathrm{~nm}$ through HGHG. There is no fresh bunch delay [10], otherwise the slice energy and radiation phase could become uncorrelated. The lack of a freshbunch delay is a significant difference from the two-stage design of FEL-2 at FERMI@Elettra [11], which has demonstrated excellent performance below $10 \mathrm{~nm}$.

The EEHG parameters are chosen to have the second energy modulation large enough and the field strength in the first chicane weak enough to keep the separation between the individual striations in longitudinal phase space well above the expected amount of energy scattering. In addition, quadrupoles are avoided until the first undulator radiating at a harmonic in order to minimize the length over which IBS can reduce the bunching at the harmonic.

The output wavelength for each beam line has been selected based on practicality: single-stage HGHG beam lines have demonstrated good performance up to the 13th harmonic [12], and the EEHG scheme has been observed in recent experiments [13] to yield significant bunching at the 15th harmonic with an energy modulation that is roughly 6 times the energy spread. The requirements for EEHG become challenging for wavelengths of the order of $1 \mathrm{~nm}$ due to tight tolerances on the seed lasers and because of the impact of energy scattering. All three beam lines have similar sensitivity to energy chirps when tuned for maximum initial bunching even though the output wavelengths are very different, because of the differences in behavior between HGHG and EEHG beam lines. The EEHG beam lines can be further adjusted to yield almost flat phase profiles for a wide variety of initial longitudinal beam distributions. The electron beam and echo parameters are given in Table I. While undulator tapering could increase the final output power, no tapering is used in these studies.

Simulations are performed using the GENESIS simulation code [14]. Chicanes are modeled by a separate code to include the effects of IBS and ISR [15]. Each dipole is treated as a region of uniform magnetic field surrounded by thin fringe fields. Simple tracking of each particle is punctuated by stochastic energy scattering events. In addition, because GENESIS does not include a model for
TABLE I. Electron beam and EEHG parameters.

\begin{tabular}{lcc}
\hline \hline Parameter & Value & Units \\
\hline Beam energy & 2.4 & $\mathrm{GeV}$ \\
Peak current & 500 & $\mathrm{~A}$ \\
Emittance & 0.6 & micron \\
Energy spread & 150 & $\mathrm{keV}$ \\
Laser power & 43 & $\mathrm{MW}$ \\
Laser wavelength & 200 & $\mathrm{~nm}$ \\
Energy modulations & 300 & $\mathrm{keV}$ \\
EEHG target wavelength & 8 & $\mathrm{~nm}$ \\
$R_{1}$ for maximum bunching & 7.45 & $\mathrm{~mm}$ \\
$R_{2}$ for maximum bunching & 0.284 & $\mathrm{~mm}$ \\
$R_{\text {echo for maximum bunching }}$ & -0.014 & $\mathrm{~mm}$ \\
\hline \hline
\end{tabular}

IBS, each macroparticle is subject to an appropriate random energy kick after the second undulator to account for IBS within the undulator. The typical IBS scattering rate is estimated at $3.0 \mathrm{keV} / \mathrm{m}^{1 / 2}$ [16]; variation of scattering rates among electrons at different transverse coordinates is ignored. The dipoles in the first echo chicane are each $0.50 \mathrm{~m}$ in length with a magnetic field of $1.28 \mathrm{~T}$; the total rms scatter due to ISR is $3.3 \mathrm{keV}$. Other chicanes are shorter with weaker fields and less ISR. The undulators are all planar, and those tuned to the seed laser have a period of $150 \mathrm{~mm}$ with 10 active periods. Other undulator sections are roughly $3.3 \mathrm{~m}$ long with $1.1 \mathrm{~m}$ breaks. The electron bunch and seed lasers are taken to have uniform current and power. Results are only shown in a 200 fs interval outside of which the energy deviations become too large for significant power to be produced (except for the HGHG beam line). Short seed lasers should produce similar results so long as the laser phases can be kept flat.

The HGHG beam line modulates the electron beam by $1.1 \mathrm{MeV}$, followed by a chicane with $R_{56}=70$ micron. The bunched beam then radiates at $20 \mathrm{~nm}$ in 5 undulator sections having a period of $50 \mathrm{~mm}$. The other two beam lines incorporate the EEHG scheme, and each seed laser generates a $300 \mathrm{keV}$ energy modulation. The design of the EEHG stage is compact to minimize IBS, and the dipole fields are kept low to minimize ISR. Bunching is produced at the 25th harmonic $(8 \mathrm{~nm})$ corresponding to $m=1$ and $p=26$. This bunching is either amplified to saturation, or upshifted again to $2 \mathrm{~nm}$ using HGHG. The radiation at $8 \mathrm{~nm}$ is produced in the same undulator sections as used for $20 \mathrm{~nm}$ but with lower magnetic fields. When going to saturation, 7 undulator sections are used. When adding an HGHG stage, 3 undulator sections at $8 \mathrm{~nm}$ are followed by a chicane with $R_{56}=1.5$ micron to yield bunching at $2 \mathrm{~nm}$. Radiation at $2 \mathrm{~nm}$ is produced in undulators with $30 \mathrm{~mm}$ period.

When tuning the EEHG beam line for flat phases the value of $R_{1}$ is decreased to $7.24 \mathrm{~mm}$ down from $7.45 \mathrm{~mm}$ for maximum bunching. This changes $R_{\text {echo }}$ from $-14 \mu \mathrm{m}$ to $-5.6 \mu \mathrm{m}$ due to the factor $m \lambda_{X} / \lambda_{1}$ in Eq. (5). The initial 


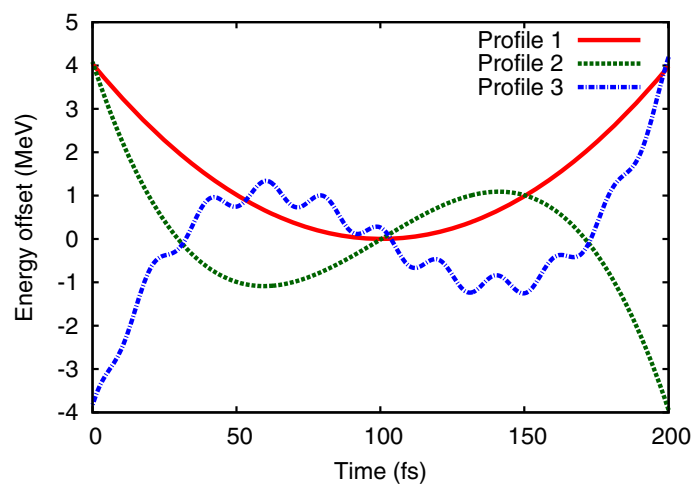

FIG. 4. Longitudinal beam profiles used in simulations of harmonic generation schemes.
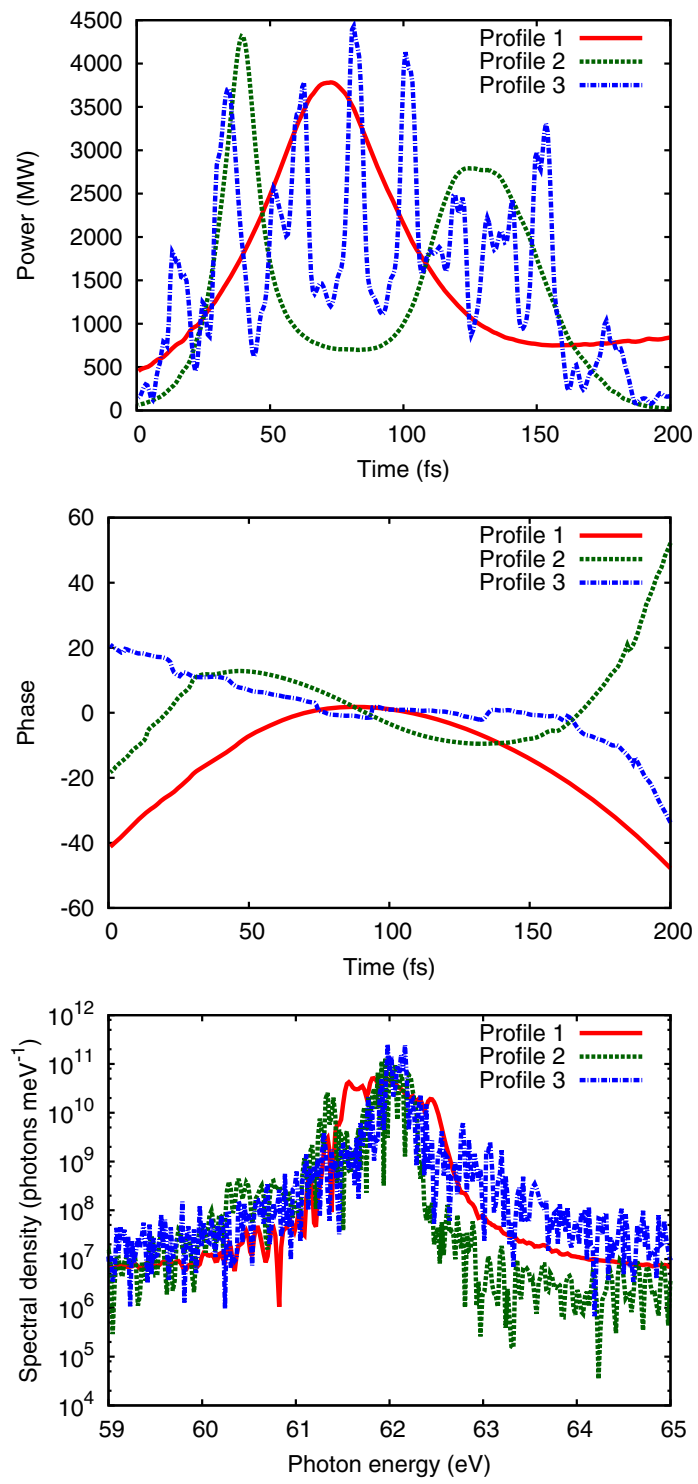

FIG. 5. Radiation characteristics at $20 \mathrm{~nm}$ after a single $\mathrm{HGHG}$ stage, showing power (top), phase (middle), and spectrum (bottom). The phases are shown as continuous functions.
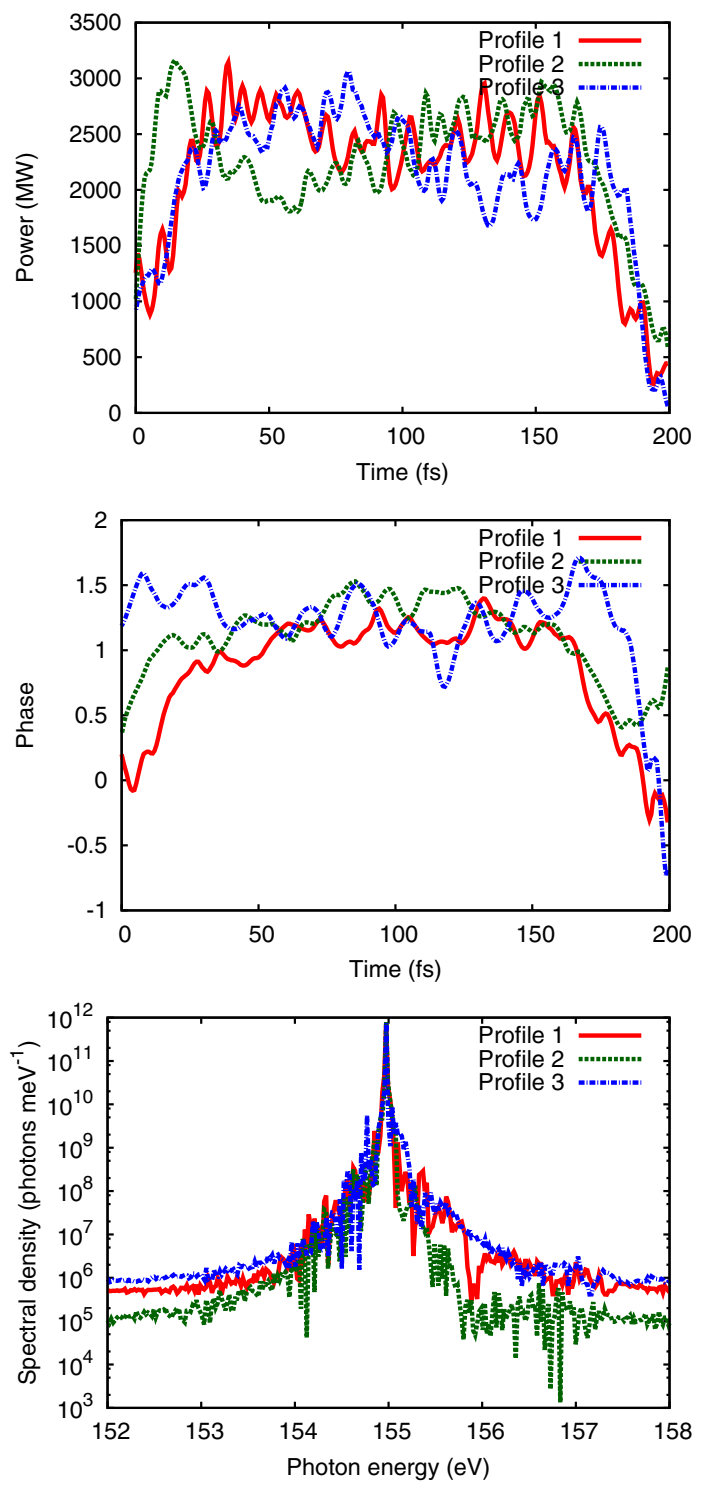

FIG. 6. Radiation characteristics at $8 \mathrm{~nm}$ at saturation, showing power (top), phase (middle), and spectrum (bottom).

bunching at $8 \mathrm{~nm}$ is thereby reduced from $5.7 \%$ to $2.9 \%$. For the combined EEHG and HGHG beam line, the corresponding numbers are $R_{1}=7.28 \mathrm{~mm}, R_{\text {echo }}=-7.2 \mu \mathrm{m}$, and $3.9 \%$ bunching. Maximum bunching with positive $R_{\text {echo }}$ is achieved when $R_{1}=6.80 \mathrm{~mm}$.

Three beam profiles are considered as extreme examples of shot-to-shot jitter in the longitudinal profile, as shown in Fig. 4. One has a quadratic energy offset, the others have cubic offsets with opposite sign. The third profile also includes a small energy modulation with a 20 -fs period to mimic the microbunching instability. The amplitude of this modulation is $\pm 250 \mathrm{keV}$, but it produces a larger phase modulation than long-wavelength energy variations $>1 \mathrm{MeV}$. This is because the slippage of the radiation field in the final undulators is enough to disrupt the correlation between local phase and local energy offset. 
Results for the beam lines are shown in Figs. 5-7. The HGHG beam line exhibits the largest phase variations despite radiating at the longest wavelength. Furthermore, portions of the beam having a large chirp radiate at wavelengths shifted significantly away from resonance and into higher order transverse modes. The output pulse energy ranges from $260 \mu \mathrm{J}$ to $319 \mu \mathrm{J}$, while a uniform beam yields $593 \mu \mathrm{J}$ over $200 \mathrm{fs}$. The drop in pulse energy is related to large frequency shifts associated with the phase variations. The only way to reduce sensitivity to energy chirps for HGHG is to increase the energy modulation and reduce the strength of the chicane.

The EEHG beam lines optimized for coherence have nearly flat phase profiles, except for profile 3 radiating at $2 \mathrm{~nm}$ where rapid phase variations are apparent.
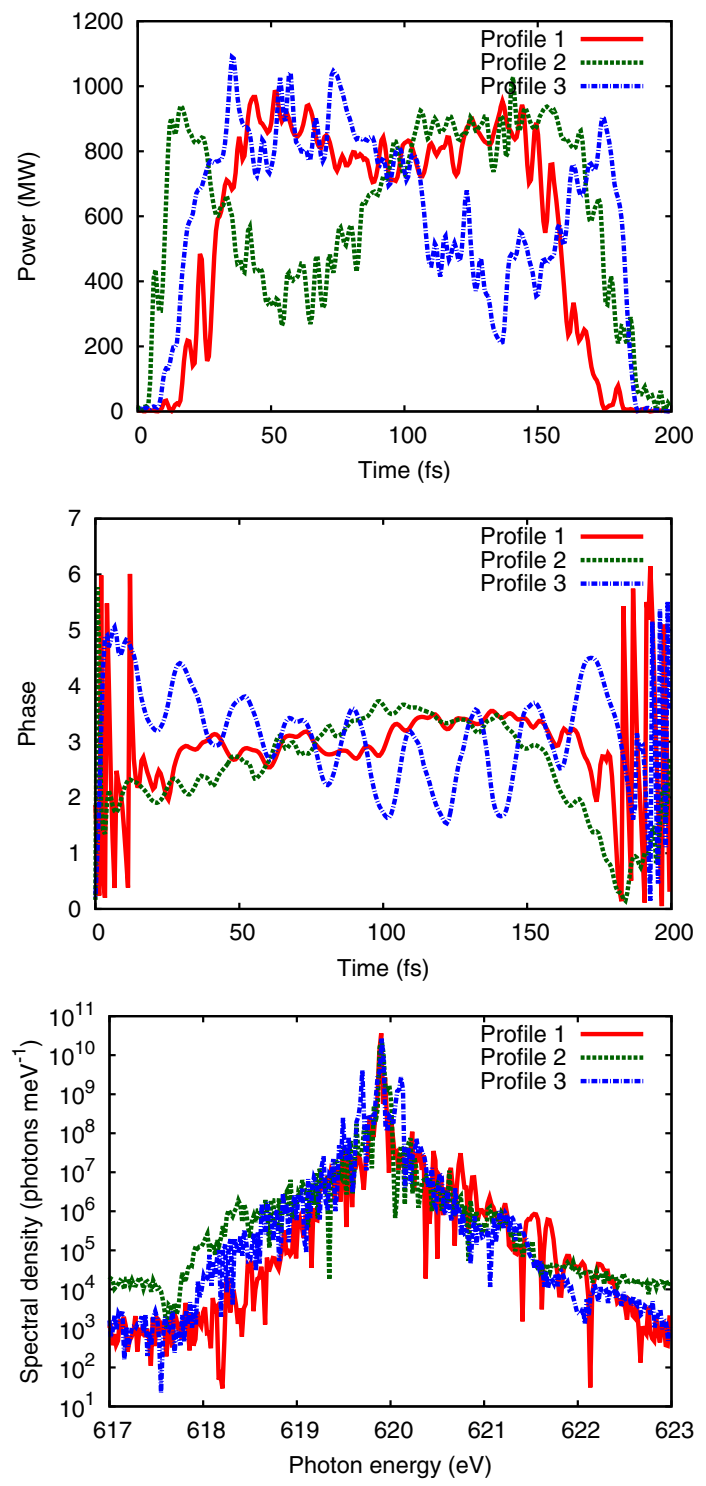

FIG. 7. Radiation characteristics at $2 \mathrm{~nm}$ at saturation, showing power (top), phase (middle), and spectrum (bottom). Phases are shown modulo $2 \pi$.
The radiation pulse does not have a flat phase profile throughout the beam line, instead the parameters are tuned to achieve optimal coherence only at the very end of the beam line. Figure 8 shows the phase profiles at $8 \mathrm{~nm}$ in the combined EEHG and HGHG beam line, just before the radiation is upshifted to $2 \mathrm{~nm}$. At this location the radiation phase mimics the initial longitudinal distribution, and the phase shifts are much larger than 1 radian. Because the phase shifts have the opposite sign from that caused by typical dispersion, by the end of the final radiation stage the phase becomes nearly flat. The main exception is for rapid energy modulations with periods comparable to the slippage length. Slippage prevents the phase shifts from exactly reversing themselves, and is the cause of the sidebands in the spectrum for profile 3 in Fig. 7, with intensity about $20 \%$ that of the central spike. In addition to changes in radiation phase and spectrum, there is also some minor variability in the total pulse energy among the different longitudinal profiles. The output pulse energy at $8 \mathrm{~nm}$ ranges from $483 \mu \mathrm{J}$ to $517 \mu \mathrm{J}$, while a uniform beam would yield $534 \mu \mathrm{J}$. The output pulse energy at $2 \mathrm{~nm}$ ranges from $121 \mu \mathrm{J}$ to $130 \mu \mathrm{J}$, while a uniform beam would yield $159 \mu \mathrm{J}$. Note that jitter in the average beam energy on the scale of the FEL parameter will have an additional impact on output power, as is common with seeded FELs. Radiation produced at $2 \mathrm{~nm}$ will be more sensitive than that at $8 \mathrm{~nm}$ to other variations in beam quality, especially peak current and emittance. This is due to both the reduced FEL parameter and the addition of an HGHG stage.

Wigner plots of the output for these three beam lines are shown in Fig. 9, in each case for profile 1 consisting of a quadratic variation in slice energy. Only the HGHG scheme yields a noticeable photon energy chirp in the output pulse. This chirp would be expected to jitter from shot to shot in the case of a fluctuating electron beam profile. Note that the vertical range of each plot covers the same absolute range in photon energy.

Finally, we consider the effect of longitudinal wakefields for the combined EEHG and HGHG beam line. The output is most sensitive to wakefields within the EEHG stage.

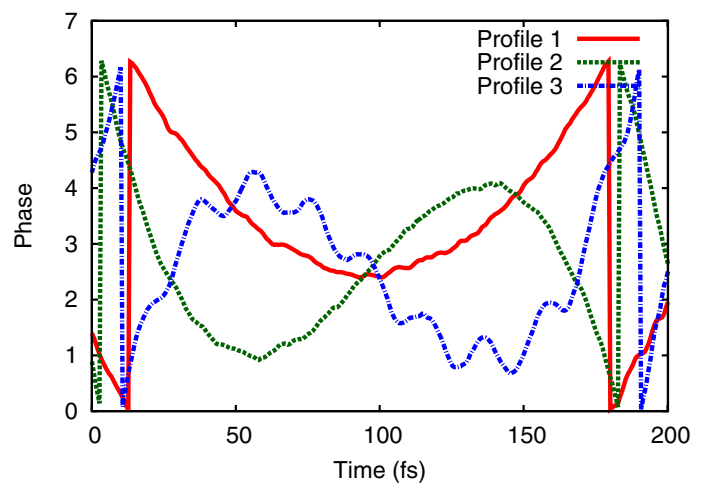

FIG. 8. Phase profiles at $8 \mathrm{~nm}$ immediately before upshifting to $2 \mathrm{~nm}$ in the combined EEHG and HGHG beam line. 

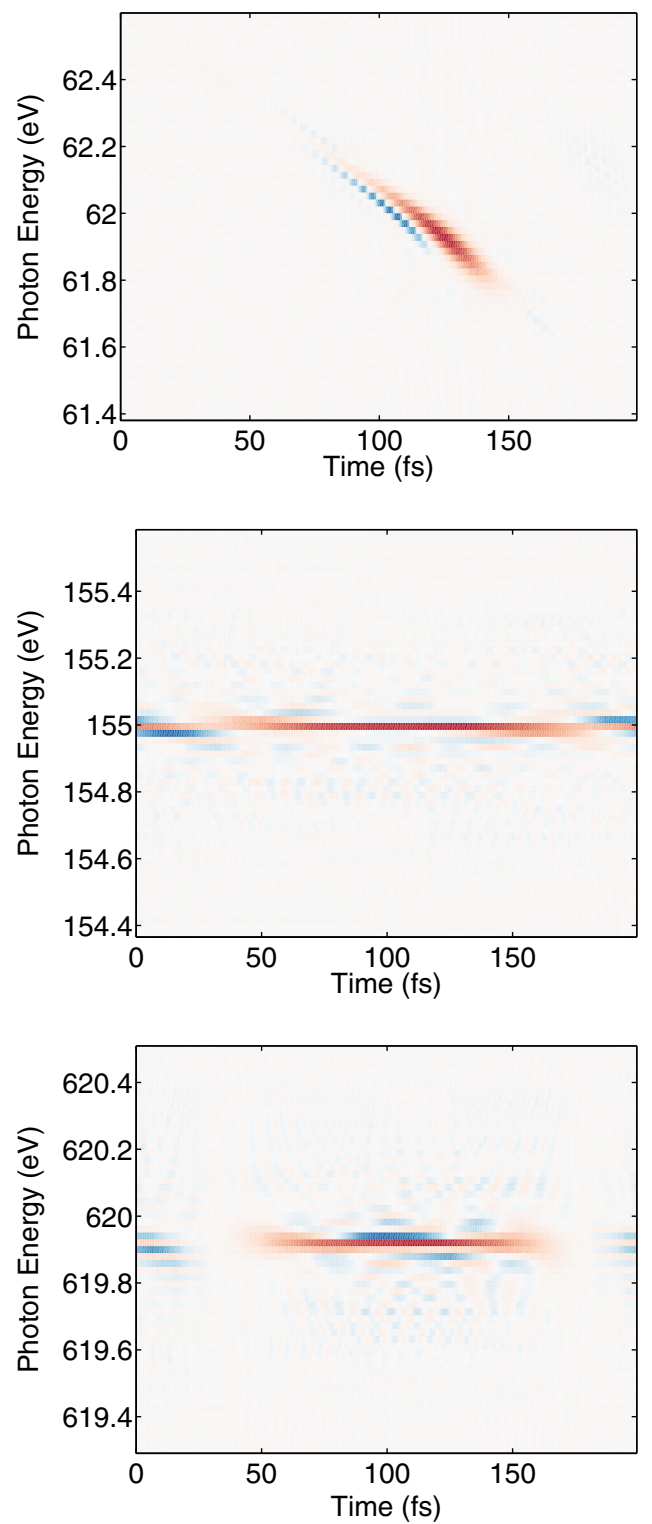

FIG. 9. Wigner plots obtained for the case of longitudinal profile 1 from the three beam lines: HGHG (top), EEHG (middle), and combined EEHG and HGHG (bottom). Red indicates a positive value, blue negative.

In the worst region, between the first two chicanes, an energy shift $\Delta \eta$ causes a shift in position of $R_{2} \Delta \eta$, and $R_{2}$ is much larger than both $R_{\text {echo }}$ and the total dispersion after the EEHG stage. We consider an artificial example where wakefields isolated between the first two chicanes generate a total energy modulation of $\pm 2 \mathrm{keV}$, with a period of $50 \mathrm{fs}$. This results in the phase varying over a range of $\approx 1.5$ radian, comparable to the phase variation for profile 3 shown in Fig. 7. For wakefields starting after the end of the EEHG stage to produce a similar phase variation would take a gradient varying between $\pm 7 \mathrm{keV} / \mathrm{m}$. These results are summarized in Fig. 10.

The design choices made to minimize ISR and IBS in the EEHG stage, such as a compact beam line and low
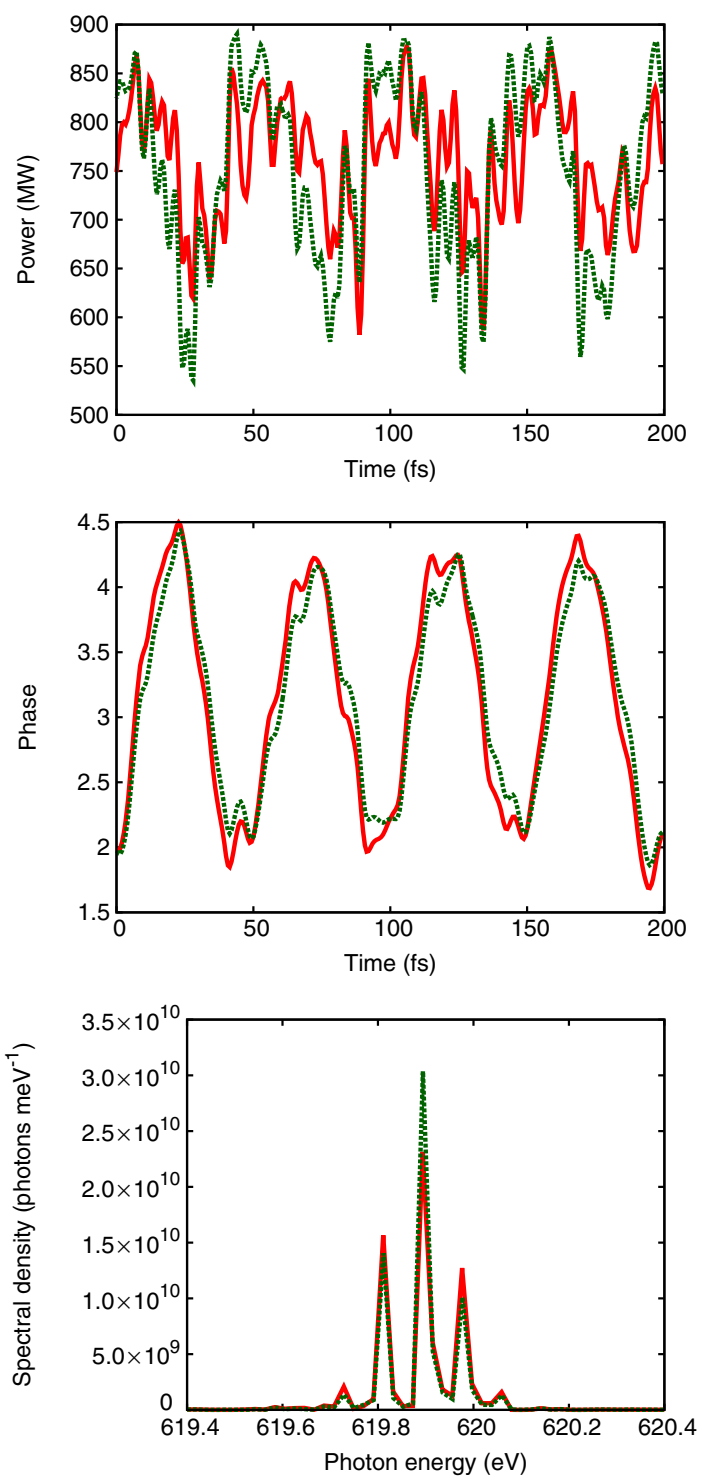

FIG. 10. Radiation characteristics for the combined EEHG and HGHG beam line of an initially flat beam subject to wakefields, showing power (top), phase (middle), and spectrum (bottom). Two separate wakefields are considered: a total energy deviation of $\pm 2 \mathrm{keV}$ induced within the EEHG stage (solid red), or a gradient ranging between $\pm 7 \mathrm{keV} / \mathrm{m}$ beginning from the end of the EEHG stage (dashed green).

magnetic fields, also help reduce wakefields from CSR and space charge. The long period for the modulating undulators should allow for a large beam pipe diameter, reducing associated wakefields. Wakefields large enough to disrupt longitudinal coherence could be generated by large current spikes, either on average or as a result of shot-to-shot jitter.

\section{CONCLUSIONS}

We have shown how to incorporate the EEHG scheme to design seeded FELs which are extremely robust to energy 
chirps and to shot-to-shot variations in the longitudinal profile. Using this scheme would allow electron beam sources which lack the capability of generating consistent, reasonably flat beams to use external lasers to produce nearly transform-limited pulses at short wavelengths. The central wavelength will also be very stable. This capability is nearly impossible to produce otherwise in the presence of large nonlinear energy chirps. Even self-seeding and oscillator schemes, which can generate short wavelengths without an external laser, are more sensitive to energy chirps than beam lines incorporating EEHG in this way. The main conventional alternatives seem to be either using specialized chicanes with the sign of $R_{56}$ reversed from the usual value or radiating in the single-spike self-amplified spontaneous emission (SASE) regime [17]. Other recently proposed schemes using beams with energy dispersion to counteract the effect of energy spread [18,19], if effective, could be used to provide similar tolerance to large chirps in the slice energy [20] at the expense of increased sensitivity to gradients in the transverse offset. The same energy integral as in Eq. (3) seems to appear in general except that in each case the coefficient scales differently. As a consequence, the sensitivity of bunching to energy spread and the sensitivity of output phase to local energy offsets are intrinsically related to each other. EEHG beam lines which are insensitive to large energy chirps could significantly increase the number of FEL facilities capable of producing coherent pulses with high photon energies. While the EEHG scheme is somewhat complicated, it also has many useful features and a great deal of flexibility.

\section{ACKNOWLEDGMENTS}

The author wishes to acknowledge Paul Emma for the realization that wakefields are an important limitation of this scheme. This work was supported by the Director, Office of Science, Office of Basic Energy Sciences, of the U.S. Department of Energy under Contract No. DE-AC02$05 \mathrm{CH} 11231$.

[1] G. Stupakov, Phys. Rev. Lett. 102, 074801 (2009).

[2] L. H. Yu, Phys. Rev. A 44, 5178 (1991).
[3] D. Xiang and G. Stupakov, Phys. Rev. ST Accel. Beams 12, 030702 (2009).

[4] Z. Huang, D. Ratner, G. Stupakov, and D. Xiang, in Proceedings of the 31st International Free Electron Laser Conference (FEL 09), Liverpool, UK (STFC Daresbury Laboratory, Warrington, 2009) p. 127, MOPC45.

[5] G. Penn, LBNL Report No. LBNL-6481E, 2013.

[6] G. Stupakov, in Proceedings of the 33rd International Free Electron Laser Conference (FEL 11), Shanghai, China (JACoW, Shanghai, China, 2011), pp. 49-52, MOPB20.

[7] G. Stupakov, in Proceedings of the 35th International Free Electron Laser Conference (FEL 13), New York, NY, USA (JACoW, New York, NY, USA, 2013), pp. 684-688, WEPS068.

[8] C. Feng, D. Wang, and Z. T. Zhao, in Proceedings of the 3rd International Particle Accelerator Conference, New Orleans, LA, 2012 (IEEE, Piscataway, NJ, 2012), p. 1724, TUPPP056.

[9] D. Ratner, A. Fry, G. Stupakov, and W. White, Phys. Rev. ST Accel. Beams 15, 030702 (2012).

[10] I. Ben-Zvi, K. M. Yang, and L. H. Yu, Nucl. Instrum. Methods Phys. Res., Sect. A 318, 726 (1992).

[11] E. Allaria, D. Castronovo, P. Cinquegrana, P. Craievich, M. D. Forno, M. B. Danailov, G. D’Auria, A. Demidovich, G. D. Ninno, S. D. Mitri, B. Diviacco, W. M. Fawley, M. Ferianis, E. Ferrari, L. Froehlich et al., Nat. Photonics 7, 913 (2013).

[12] E. Allaria, R. Appio, L. Badano, W. A. Barletta, S. Bassanese, S. G. Biedron, A. Borga, E. Busetto, D. Castronovo, P. Cinquegrana, S. Cleva, D. Cocco, M. Cornacchia, P. Craievich, I. Cudin et al., Nat. Photonics 6, 699 (2012).

[13] E. Hemsing, M. Dunning, C. Hast, T. O. Raubenheimer, S. Weathersby, and D. Xiang, Phys. Rev. ST Accel. Beams 17, 070702 (2014).

[14] S. Reiche, Nucl. Instrum. Methods Phys. Res., Sect. A 429, 243 (1999).

[15] G. Penn, LBNL Report No. LBNL-6762E, 2014.

[16] Z. Huang, SLAC Report No. SLAC-TN-05-026, 2002.

[17] R. Bonifacio, L. DeSalvo, P. Pierini, N. Piovella, and C. Pellegrini, Phys. Rev. Lett. 73, 70 (1994).

[18] H. Deng and C. Feng, Phys. Rev. Lett. 111, 084801 (2013).

[19] C. Feng, T. Zhang, H. Deng, and Z. Zhao, Phys. Rev. ST Accel. Beams 17, 070701 (2014).

[20] G. Wang, C. Feng, H. Deng, T. Zhang, and D. Wang, Nucl. Instrum. Methods Phys. Res., Sect. A 753, 56 (2014). 